\title{
Conditions under which amobarbital sodium influences contrast in consummatory behavior
}

\author{
CHARLES F. FLAHERTY, HOWARD C. BECKER, and CYNTHIA DRISCOLL \\ Rutgers-The State University, New Brunswick, New Jersey
}

\begin{abstract}
The effect of amobarbital sodium (ABS) on -contrast effects in consummatory behavior was investigated in three experiments. In Experiment 1, rats were shifted from $32 \%$ sucrose to $4 \%$ sucrose after 11 days' experience with the $32 \%$ solution. ABS $(15,17.5$, and $20 \mathrm{mg} / \mathrm{kg})$ was administered during the final three preshift days and throughout the postshift period. Contrast was not reduced by this treatment, and, in fact, the higher doses tended to retard recovery from contrast. In Experiment 2, ABS $(17.5 \mathrm{mg} / \mathrm{kg})$ was injected on the 1st and 2nd postshift days only. Under these administration conditions, the drug reduced degree of contrast. In Experiment $3, A B S(12,17.5,22 \mathrm{mg} / \mathrm{kg})$ tended to enhance the degree of simultaneous negative contrast that occurred when rats were repeatedly shifted between $32 \%$ and $4 \%$ sucrose. Thus, the conditions under which ABS might be expected to reduce contrast in consummatory behavior are restricted. Similarities and differences in the effects of ABS, chlordiazepoxide, and ethanol on contrast are discussed.
\end{abstract}

The consummatory behavior of rats given experience with two different sucrose solutions differs from that of rats given access to a single concentration of sucrose. For example, animals given $5 \mathrm{~min}$ access to $32 \%$ sucrose for a number of days and then shifted to $4 \%$ sucrose consume substantially less of the $4 \%$ than do animals given only the $4 \%$ sucrose all along. This result, termed a successive negative contrast effect, has been shown to be sensitive to the effects of chlordiazepoxide (CDP), but under limited conditions; injections of CDP during both preshift and postshift, or on the 1st postshift day, have not been found to affect contrast, but injections on the 2 nd or 3rd postshift day eliminate contrast (Flaherty, Lombardi, Wrightson, \& Deptula, 1980; Vogel \& Principi, 1971). Scopolamine appears to have no tendency to reduce this type of contrast; instead, it tends to have a general effect of decreasing lick rate for $4 \%$ sucrose (Flaherty \& Meinrath, 1979).

A second type of contrast in consummatory behavior is obtained when rats are given repeated, brief access periods to two tubes containing different sucrose solutions. For example, rats given alternating 1-min access periods to tubes containing either $32 \%$ or $4 \%$ sucrose will lick more for $32 \%$ sucrose when the alternative tube contains $4 \%$ than when both tubes contain $32 \%$ (positive contrast) and less for the $4 \%$ when the alternative tube contains $32 \%$ than when both contain $4 \%$ (negative contrast). These effects,

Correspondence may be addressed to C. F. Flaherty, Department of Psychology, Busch Campus, Rutgers University, New Brunswick, New Jersey 08903.

-Article accepted by previous editor, Richard F. Thompson. termed simultaneous contrast effects, are found both when the same animals serve under contrast and control conditions (on different days) and when separate groups are used as controls (Flaherty \& Largen, 1975). These simultaneous contrast effects seem to be insensitive to a variety of drugs, including CDP (Flaherty, Lombardi, Kapust, \& D'Amato, 1977; Flaherty, Wrightson, Deptula, \& Duston, 1979), imipramine (Flaherty et al., 1977), and scopolamine (Flaherty \& Meinrath, 1979).

The present experiment is concerned with the effects of amobarbital sodium (ABS) on both types of contrast obtained in the consumption of sucrose solutions. ABS has been previously shown to influence contrast obtained in instrumental tasks with food reward. For example, ABS prevented the successive negative contrast effect that normally occurs when rats are shifted from a 15-pellet reward to a single-pellet reward in a runway (Rosen, Glass, \& Ison, 1967), and Ridgers and Gray (1973) found that amylobarbitone eliminated a negative contrast effect that occurred in operant behavior following the insertion of a probe stimulus that signaled a lower frequency of reinforcement than that available on the prevailing schedule. Interestingly, amylobarbitone seemed to have no effect on a positive contrast effect produced by the insertion of a probe stimulus signaling the availability of a higher rate of reinforcement (Ridgers \& Gray, 1973).

ABS has been investigated in other situations in which it has been found to (1) increase approach behavior in the presence of stimuli signaling shock (Barry \& Miller, 1962), (2) increase resistance to extinction (Barry, Wagner, \& Miller, 1962), and (3) decrease running times for electrical stimulation in vari- 
ous limbic sites (Reid, Wasden, \& Courtney, 1970). All of these effects, as well as others (e.g., Miller, 1961), could be attributed to fear- or frustrationreducing properties of the drug. Such an interpretation might also apply to the effect of ABS on negative contrast.

In an earlier paper (Flaherty \& Driscoll, 1980), we presented evidence that ABS reduced, but did not eliminate, successive negative contrast when injected on either the 1st or 2 nd postshift day. These results contrasted with those obtained with chlordiazepoxide, which was not effective on the 1st postshift day. In the present paper, we present evidence from three experiments concerned with the effects of ABS on (1) successive negative contrast following a shift in sucrose solutions when the drug was administered both during preshift and postshift periods, (2) on successive negative contrast when the drug was administered on the first and second postshift day only, and (3) on simultaneous positive and negative contrast.

\section{EXPERIMENT 1}

In this experiment, two groups of rats were given 11 days of access to either $32 \%$ or $4 \%$ sucrose prior to the shift of the former $32 \%$ group to the $4 \%$ solution. On each of the last 3 preshift days and during the first 3 postshift days, the animals were injected with either saline or ABS. One-fourth of the animals were injected with saline, one-fourth with $15 \mathrm{mg} / \mathrm{kg}$ ABS, one-fourth with $17.5 \mathrm{mg} / \mathrm{kg} \mathrm{ABS}$, and onefourth with $20 \mathrm{mg} / \mathrm{kg} \mathrm{ABS}$. These doses overlap the values found by Ridgers and Gray (1973) and by Rosen et al. (1967) to eliminate contrast.

\section{Method}

Subjects. Forty-eight adult male Sprague-Dawley-derived rats were used as subjects. The rats were deprived to $82 \%$ of their freefeeding body weights and maintained at that level throughout the experiment by once-a-day feeding. All animals were individually housed with free access to water and maintained under a 14/10 light-dark cycle.

Apparatus. Testing was conducted in six identical metal cages measuring $24.5 \times 17.5 \times 18 \mathrm{~cm}$. On one side of each cage was a centrally located 1 -cm-diam hole, $7 \mathrm{~mm}$ above the wire-mesh floor. Located outside the cage was a graduated drinking tube positioned so that the orifice of the drinking tube was centered in the hole, flush with the outside wall of the chamber. A contactrelay circuit was used to measure the licking response.

Procedure. The subjects were assigned to one of eight groups $(n=6)$ matched for body weight. On 2 successive days immediately prior to the first preshift day, the rats were preexposed to sucrose in the home cage. Four of the groups received a $32 \%$ sucrose solution, while the remaining four groups received a $4 \%$ sucrose solution. On subsequent days, the rats were placed into the apparatus for a 5-min period starting from the time of the first lick. Half of the animals received $32 \%$ and half $4 \%$ sucrose during the 11-day preshift period.

On the 9th day preshift, as well as all subsequent test days, onefourth of the animals in each sucrose group were injected with $15 \mathrm{mg} / \mathrm{kg}$, one-fourth with $17.5 \mathrm{mg} / \mathrm{kg}$, one-fourth with $20 \mathrm{mg} / \mathrm{kg}$ ABS, and one-fourth with an approximate volume equivalent of physiological saline (amobarbital sodium purchased from Ganes Chemicals, New York, N.Y.). The drugs were mixed immediately prior to the start of each test session with physiological saline on a $\mathrm{mg} / \mathrm{kg}$ basis. The injections were given ip $10 \mathrm{~min}$ prior to the start of each test session.

During the postshift phase (Days 12-15), all animals received a $4 \%$ sucrose solution. The number of licks made during both preshift and postshift periods was recorded.

The sucrose solutions were prepared by weight from commercialgrade sugar and tap water. The solutions were mixed daily, $24 \mathrm{~h}$ prior to each test session and presented at room temperature.

\section{Results}

Preshift. During the preshift period, the animals given access to $32 \%$ sucrose licked more than the animals given $4 \%$, but this difference diminished over the preshift period and was not statistically reliable over the last 3 preshift days $[F(1,37)=3.21, p=.08]$. Injection of the drug for the first time (Day 9 preshift) had a slight debilitating effect on consummatory behavior; the 17.5- and $20-\mathrm{mg} / \mathrm{kg}$ ABS groups licked at a lower rate than the $15-\mathrm{mg} / \mathrm{kg}$ group, but the lick rate of only the $17.5-\mathrm{mg} / \mathrm{kg}$ group was reliably below that of saline controls [drug $\times$ day, $F(6,73)=2.65$, $\mathrm{p}<.05$, followed by Fisher's least significant difference (LSD) tests]. By the last preshift day, the drug was having a slight energizing effect on consummatory behavior; the $15-$ and $20-\mathrm{mg} / \mathrm{kg}$ groups licked more than did the saline group, and the $20-\mathrm{mg} / \mathrm{kg}$ group licked more than the $17.5-\mathrm{mg} / \mathrm{kg}$ group (LSD tests, $\mathrm{p}=.05$ ).

Postshift. The postshift lick frequencies (presented in Figure 1) indicated a large contrast effect in that the shifted animals licked reliably less than unshifted controls $(F(1,37)=38.30, p<.001)$. In addition, there were variations in degree of contrast as a function of drug condition across the 4 postshift days $[\mathrm{F}(3,109)=2.33, \mathrm{p}<.05]$. Analysis of this interaction

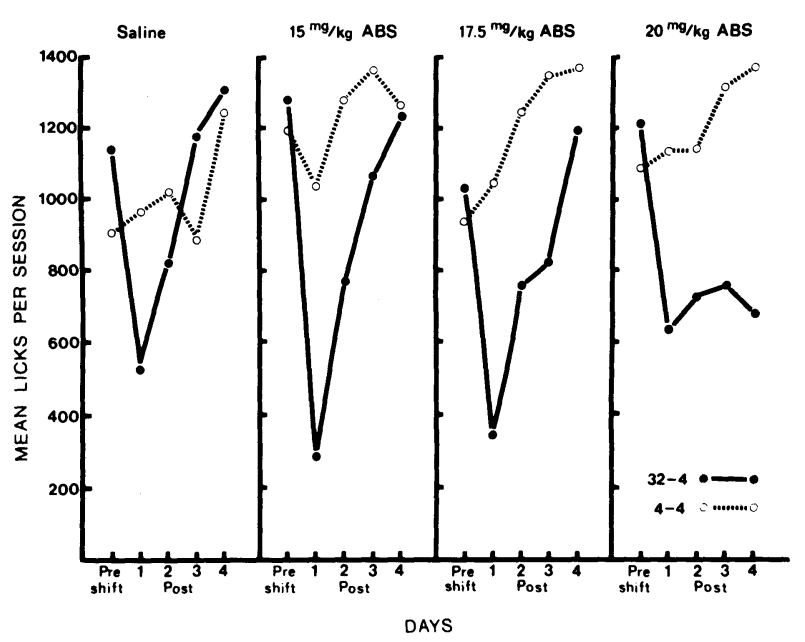

Figure 1. Mean preshift ("Pre') and postshift licks for sucrose as a function of contrast and drug conditions. The values for "Pre" represent the mean of the last 3 preshift days. 
with the LSD test indicated the following pattern of results: Contrast was reliable in the saline injected animals only on the 1st postshift day, in the $15-\mathrm{mg} / \mathrm{kg}$ ABS rats on the first 2 postshift days, in the $17.5-\mathrm{mg} / \mathrm{kg}$ group on the first 3 days, and in the $20-\mathrm{mg} / \mathrm{kg}$ group on all 4 postshift days.

Differences in the unshifted animals as a function of drug condition are apparent in Figure 1: the lickfrequency for the $4 \%$ solution is higher in all three drug groups than in the saline injected controls. However, these differences were reliable only on the 3 rd postshift day ( $p=.05$, LSD test).

\section{Discussion}

It is apparent that ABS did not reduce the degree of negative contrast under the conditions of this experiment. The effects of the drug were small, but they tended to prolong the period of contrast rather than reduce it. This was particularly true with the $20-\mathrm{mg} / \mathrm{kg}$ dose, with which there was no indication of recovery from the effects of the shift. In fact, it is our interpretation that the effect of the $20-\mathrm{mg} / \mathrm{kg}$ dose represents the only substantial effect of the drug on recovery from contrast. The apparent retardation in recovery obtained with the two lower doses was slight and the recovery of the saline group was more rapid than usually occurs in these experiments. In fact, untreated animals usually show reliable contrast for 2 postshift days and, occasionally, for 3 or more postshift days (Flaherty et al., 1980; Lombardi \& Flaherty, 1978). Thus, we would not weigh too importantly the effects of the $15-$ and $17.5-\mathrm{mg} / \mathrm{kg}$ doses on recovery from contrast.

Observations of the animals indicated that the reduced rate of recovery was not due to a sedative effect of the drug. Rather, the drug-injected animals appeared to be particularly agitated and spent a good deal of time apparently attempting to escape from the apparatus.

The failure of ABS to reduce contrast in this experiment is consistent with a similar finding for chlordiazepoxide (CDP), which resulted in the same consummatory response paradigm (Flaherty et al., 1980). However, CDP has been found to reduce such consummatory contrast when the first injection of the drug occurred on the 2nd postshift day. Injections of CDP on the 1st postshift day were without effect (Flaherty et al., 1980; Vogel \& Principi, 1971). We had previously found that ABS also reduced contrast when the first injection was made on the 2 nd postshift day, but, unlike CDP, ABS was also effective when administered on the 1st postshift day (Flaherty $\&$ Driscoll, 1980). Thus, there are two similarities and one difference in the effects of CDP and ABS on consummatory contrast: both are ineffective in reducing contrast when administered preshift and postshift, both do reduce contrast when administered for the first time on the 2 nd postshift day, and, finally,
ABS, but not CDP, seems also to be effective in reducing contrast on the 1st postshift day.

A second experiment was conducted in order to replicate the earlier finding that ABS reduced contrast when administered on Postshift Day 1.

\section{EXPERIMENT 2}

In this experiment, rats were injected with $17.5 \mathrm{mg} /$ $\mathrm{kg} \mathrm{ABS}$ on the first 2 postshift days. This dose was effective in reducing contrast when administered on either the 1st or 2nd postshift days (Flaherty \& Driscoll, 1980), but did not reduce contrast when administered both preshift and postshift in Experiment 1.

\section{Method}

Subjects. Sixteen male naive rats of the Sprague-Dawley strain were used as subjects. The rats (80-85 days old) were individually housed on a 14/10 L/D cycle and maintained at $82 \%$ of their freefeeding body weights throughout the experiment. Water was available ad lib.

Apparatus. The subjects were tested in four identical metal cages $(24.5 \times 17.5 \times 18 \mathrm{~cm})$. On one side of each cage, a $1-\mathrm{cm}$ diam hole was centrally located $7 \mathrm{~mm}$ above the floor. A graduated cylinder was placed outside the chamber such that the orifice of the drinking spout was centered in the hole flush with the outside wall of the cage. The licking response was recorded by a contact-relay circuit.

Procedure. The subjects were divided randomly into four groups $(n=4)$. Half the animals received a $32 \%$ sucrose solution for 10 (preshift) days and then $4 \%$ sucrose for 3 (postshift) days. The remaining animals served as unshifted controls, receiving the $4 \%$ solution on all 13 days. The animals were allowed access to the sucrose solutions for 5 min every day, timed from the first lick.

On Postshift Days 1 and 2 (Days 11 and 12), 17.5-mg/kg injections of amobarbital sodium (ABS) were administered to half of the shifted and half of the unshifted animals $10 \mathrm{~min}$ prior to the start of the sessions. The other groups of four shifted and four unshifted animals received injections of an equivalent volume of physiological saline on the first 2 postshift days. All injections were ip, with no injections being administered on the 3rd postshift day.

The ABS was obtained from Ganes Chemicals (New York, N.Y.) and was mixed with physiological saline immediately prior to each test session. Sucrose solutions were prepared by weight from commercial-grade cane sugar and tap water. The solutions were prepared daily, $24 \mathrm{~h}$ before each session.

\section{Results}

The results are expressed in terms of mean licks per 5-min session and mean lick proportions (postshift/ terminal preshift + postshift).

The data indicate that animals shifted from $32 \%$ to $4 \%$ consumed less $4 \%$ solution than did animals maintained on $4 \%$ all along. This was supported by an analysis of variance in which the sucrose concentration $\times$ drug $\times$ day interaction was reliable for both the licks and proportions analyses $[\mathrm{F}(2,36)=$ $4.28, \mathrm{p}<.05$, and $\mathrm{F}(2,36)=4.59$, $\mathrm{p}<.05$, respectively]. Subsequent analysis with Fisher's LSD test revealed that saline animals showed a reliable negative contrast effect for all 3 postshift days. This was also true for the licks and proportions analyses. Sub- 


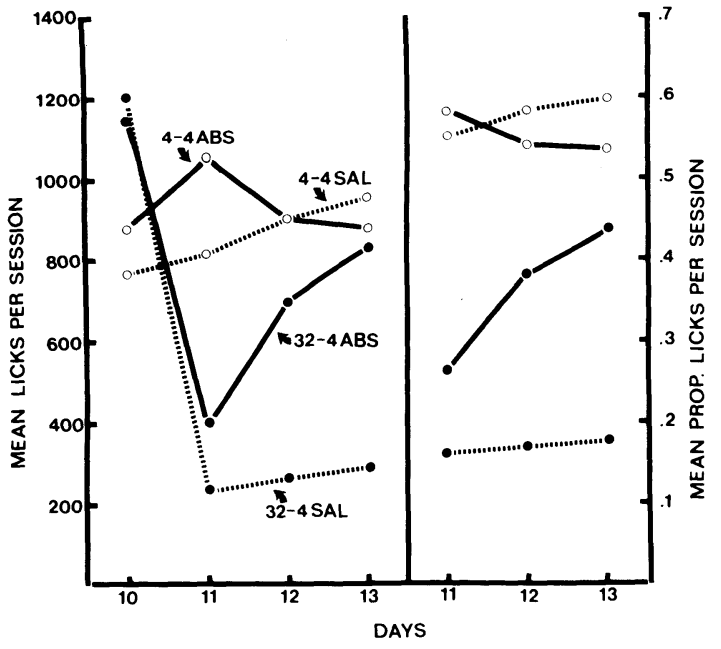

Figure 2. Left panel: Mean preshift and postshift licks for sucrose as a function of shift and drug conditions. The ABS animals were injected on the 1st and 2nd postshift days. Right panel: The same postshift data expressed as a proportion of preshift lick frequency.

jects injected with ABS showed a significant contrast effect on the 1st postshift day only. Using proportions as the dependent variable, ABS animals showed a reliable contrast effect on the 1 st and 2 nd postshift days.

The increased recovery rate from contrast of the drug animals was further supported by the fact that the ABS-shifted animals licked reliably more than the saline-shifted animals on the 2 nd and 3 rd postshift days. This was true of the licks and proportions analyses. Lick rates and proportions on the 1st postshift day were greater for the drug than the saline animals, but this effect was not reliable.

\section{Discussion}

The results of the first two experiments indicate that ABS is effective in reducing contrast when it is administered during the postshift period only, but that it does not reduce contrast when administered both preshift and postshift. The results of Experiment 2 differed somewhat from those of a similar experiment published earlier (Flaherty \& Driscoll, 1980). In the earlier paper, it was found that ABS produced a reliable reduction in contrast when administered on either the 1st or 2nd postshift day. In Experiment 2, there was a reduction in contrast on the 1st day of drug administration, but the effect was not statistically reliable. When the drug was administered again on the 2nd postshift day, contrast was very substantially reduced. Thus, ABS and CDP are similarly effective in producing a large reduction in contrast on the 2 nd postshift day. The effects on the 1st postshift day remain somewhat equivocal, but ABS seems more likely to be effective on Day 1 than CDP (Flaherty \& Driscoll, 1980, Experiment 2; Flaherty et al., 1980).
Both ABS and CDP reduce contrast in runway behavior when the number of food pellets is reduced (Rosen et al., 1967; Rosen \& Tessel, 1970). These reductions occurred under conditions in which the drugs were administered during both the preshift and postshift periods. The present data obtained with ABS and the earlier data obtained with CDP (Flaherty et al., 1980) show that this procedure is ineffective in reducing contrast in consummatory behavior produced by shifts in sugar solutions. The limitation of the effectiveness of these drugs in consummatory contrast to the condition in which the drug is administered for the first time during the postshift period may indicate that the contrast, and effect of the drugs in reducing contrast, is not operating through the same mechanisms in the runway and consummatory response paradigms. Speculation regarding these potentially different mechanisms should perhaps be restrained until additional data is available.

\section{EXPERIMENT 3}

A third experiment was conducted in order to further investigate the similarity in the effects of ABS and CDP in contrast produced by shifts in sucrose solutions. Rats given repeated, alternating access to $32 \%$ and $4 \%$ sucrose drink less of the $4 \%$ sucrose than do animals that experience only the $4 \%$ solution. These "simultaneous" contrast effects are long lasting, apparently enduring as long as the concentration differences are present, and are unaffected by CDP in a variety of test conditions and under a variety of doses (Flaherty et al., 1977; Flaherty et al., 1980; Flaherty et al., 1979). In the present experiment, we examined the effects of ABS $(12,17.5$, and $22 \mathrm{mg} / \mathrm{kg})$ on contrast obtained in this simultaneous paradigm.

\section{Method}

Subjects. Eight Sprague-Dawley-derived rats purchased from Charles River Breeding Farms were used as subjects. These rats had had prior experience in a successive contrast experiment. Four of the rats had had prior experience with both $32 \%$ and $4 \%$ sucrose solutions (i.e., were shifted animals), and the other four rats had had prior $4 \%$ experience only. No animals had previously been injected. Maintenance and housing were as in the previous experiment.

Apparatus. Testing was conducted in a Plexiglas chamber measuring $30 \times 25 \times 25 \mathrm{~cm}$. On one side of the chamber were two centrally located $1.5-\mathrm{cm}$-diam holes spaced $21.7 \mathrm{~cm}$ apart and $4 \mathrm{~cm}$ above the wire mesh floor. Two graduated drinking tubes, located outside the chamber, were programmed so that either tube could be automatically moved into a drinking position in which the orifice of the drinking spout was centered in the $1.5-\mathrm{cm}$-diam hole, flush with the outside wall of the chamber. Pilot lights, mounted on either side of the chamber near the drinking access holes, were illuminated whenever the tube in closer proximity to that light was in the drinking position. A contact-relay circuit was used to measure the licking response.

Procedure. For 2 successive days prior to the beginning of the test sessions, the rats were placed in the apparatus for $5 \mathrm{~min}$ each day to familiarize them with the chamber. On each test day, the 
rats were placed in the chamber with the left tube in the drinking position. The tube remained available for a $45-\mathrm{sec}$ period starting from the time of the first lick. At the termination of this period, the left tube retracted and the right tube immediately moved into position for $45 \mathrm{sec}$ following the first lick. The right tube then retracted and the left tube moved into position. This procedure continued for a total of three presentations of each tube. If the rat made no response within $60 \mathrm{sec}$, the tube automatically retracted and the alternate tube moved into position.

The sucrose solutions contained in the tubes were varied across days in 4-day cycles. On 2 days of each cycle, both tubes contained the same solution, $32 \%$ on one day and $4 \%$ on the other day. On the remaining 2 days, one bottle contained the $32 \%$ solution and the other the $4 \%$ solution. On one of these days, the $32 \%$ solution was in the left bottle, and on the other, it was in the right bottle. Within each 4-day cycle, the sequence of sucrose conditions was $32 \%$ in the left and $4 \%$ in the right (32-4), 32-32, 4-32, and 4-4. This sequence was repeated four times, for a total of 16 days of testing.

In different 4-day sucrose cycles, the animals were injected with either saline or amobarbital sodium in doses of 12, 17.5, and $22 \mathrm{mg} / \mathrm{kg}$. Drug administration was varied according to a randomly selected $4 \times 4$ Latin-square plan, with a 2 -day break in testing between each drug condition. Thus, both sucrose condition and drug treatment were administered within subjects. Since the eight subjects differed in their prior experience with sucrose, this factor constituted a variable orthogonal to the drug and sucrose variables. Four subjects had a prior history of experience with $4 \%$ only, and four had had prior experience with both $32 \%$ and $4 \%$ sucrose. Thus, prior history was a between-subjects factor.

The number of licks was recorded for each $45-\mathrm{sec}$ period, as was the latency of the first lick made after the bottles switched. For purposes of analysis, the licks made to the left and right tubes under identical sucrose conditions were averaged together. For example, when both tubes contained $32 \%$ sucrose, a mean of the licks made on the right and left tubes was obtained for each $45-\mathrm{sec}$ access period. This procedure yielded three numbers for each session for each rat: means for the first, second, and third access periods to the left and right tubes. Similar procedures were used for the $4 \%$ vs. $4 \%$ sucrose condition. When the tubes contained different solutions, the means were taken across days. Thus, the lick rate for the first access period to $4 \%$ on the left when the right tube contained $32 \%$ was averaged with the lick rate for the first access period to $4 \%$ on the right when the left tube contained $32 \%$. This procedure yielded three means, one for each access period, representing licking for $4 \%$ when the alternative tube contained $32 \%$, and three means representing licking for $32 \%$ when the alternative tube contained $4 \%$.

\section{Results}

Separate analyses of variance were conducted for positive and negative contrast in each 45 -sec access period. Examination of the animals' lick rates for $32 \%$ when the alternative was $4 \%$ as compared with when both tubes contained $32 \%$ indicated that the rats tended to lick more for $32 \%$ under the $32 \%$ vs. $4 \%$ condition than under the $32 \%$ vs. $32 \%$ condi- tion. However, this apparent positive contrast was not statistically reliable [in the third access period, when the differences were greatest, $F(1,6)=5.78$, $.05<\mathrm{p}<.10]$. There was no indication of a reliable positive contrast effect in the first two access periods. The effects of the drug, both in isolation and in interaction with sucrose concentration and prior history, did not approach statistical reliability in the analysis of the positive contrast data.

Analysis of the negative contrast data indicated no reliable effects of any of the variables in the first access period. In the second access period, there was an overall group effect $[F(1,6)=10.04, p<.05]$, which indicated a higher lick rate in the animals with a prior history of consuming $4 \%$ only. There was also a reliable negative contrast effect in this access period; that is, the rats drank less of the $4 \%$ when the alternative tube contained $32 \%$ than they did when both tubes contained $4 \%[F(1,6)=9.50, p<.05]$. Finally, there was a reliable drug $\times$ concentration interaction $[F(3,18)=6.45, p<.01]$. Information relevant for the interpretation of this interaction is presented for Access Period 2 in Table 1. Examination of these data shows that lick rate for $4 \%$ sucrose under noncontrast conditions (4-4) increased as drug dose increased, with the difference between the saline and $22-\mathrm{mg} / \mathrm{kg}$ levels being reliable by LSD test $(\mathrm{p}=.05)$. Under contrast conditions (4 vs. 32 ), there is less of a systematic effect of the drug, with the lowest lick rates occurring under the highest drug level (but, by LSD test, these are not reliably different from those occurring at other drug levels). Thus, this interaction indicates that contrast is increasing in size as drug dose increases, but the mechanism of the increased contrast is principally a greater lick rate under control conditions. There were no other reliable effects occurring in the second access period.

Analysis of the negative contrast data for the third access period again indicated a reliable group effect $[F(1,6)=13.05, p<.05]$, reflecting the continued higher lick rate in the animals with previous $4 \%$ experience only. There was also an overall negative contrast effect $[F(1,6)=13.05, p<.05]$. The interaction between contrast and drug was no longer reliable $[F(3,18)=1.70, p>.10]$, although a similar trend was still present in the data (see Table 1).

In summary, both positive and negative contrast tended to develop as the animals had repeated access

Table 1

Mean Lick Rates in the Second and Third Access Periods as a Function of Sucrose and Drug Conditions

\begin{tabular}{|c|c|c|c|c|c|c|c|c|}
\hline \multirow{3}{*}{$\begin{array}{l}\text { Access } \\
\text { Period } \\
\end{array}$} & & & \multicolumn{6}{|c|}{ Sucrose Condition } \\
\hline & \multicolumn{2}{|c|}{ Saline } & \multicolumn{2}{|c|}{$12 \mathrm{mg} / \mathrm{kg}$} & \multicolumn{2}{|c|}{$17.5 \mathrm{mg} / \mathrm{kg}$} & \multicolumn{2}{|c|}{$22 \mathrm{mg} / \mathrm{kg}$} \\
\hline & 4 vs. 4 & 4 vs. 32 & 4 vs. 4 & 4 vs. 32 & 4 vs. 4 & 4 vs. 32 & 4 vs. 4 & 4 vs. 32 \\
\hline $\begin{array}{l}2 \\
3\end{array}$ & $\begin{array}{r}99.25 \\
102.13\end{array}$ & $\begin{array}{l}83.25 \\
94.50\end{array}$ & $\begin{array}{r}99.62 \\
111.50\end{array}$ & $\begin{array}{r}103.00 \\
93.30\end{array}$ & $\begin{array}{l}114.12 \\
127.63\end{array}$ & $\begin{array}{l}78.62 \\
79.75\end{array}$ & $\begin{array}{l}145.75 \\
127.25\end{array}$ & $\begin{array}{l}55.75 \\
77.25\end{array}$ \\
\hline
\end{tabular}


to the solutions; negative contrast was more reliable than positive contrast; ABS seemed to enhance the development of negative contrast, but primarily by increasing lick rates under control conditions.

The development of contrast over repeated access periods that occurred in the present study is a general finding in these simultaneous contrast studies (e.g., Flaherty \& Largen, 1975). The failure of positive contrast to reach statistical reliability is unusual in this paradigm (e.g., Flaherty \& Largen, 1975; Flaherty et al., 1977) and may reflect the fact that all animals used in this study had had prior licking experience. The effect this prior experience had in reliably increasing the lick rates in rats with 13 days of prior $4 \%$ experience, as compared with those with only 5 days of prior $4 \%$ experience (as well as 8 days of prior $32 \%$ experience), may reflect the development of a licking "habit" under the control of the particular sucrose concentration.

Amobarbital sodium had no overall effect on negative contrast but did tend to enhance lick rates for $4 \%$ sucrose under control conditions ( $4 \%$ vs. $4 \%)$, thereby also enhancing negative contrast. This effect of the drug was more pronounced in the second access period than in the third. There was no reliable tendency of the drug to reduce contrast under any of the conditions of the experiment.

\section{GENERAL DISCUSSION}

Previous studies have shown that CDP, in various doses, does not influence the degree of simultaneous negative contrast, under both within-subject and between-subject test conditions and both when administered early and late in contrast training (Flaherty et al., 1977; Flaherty et al., 1979). The present Experiment 3 indicates that ABS also does not reduce the degree of simultaneous negative contrast. In fact, the only tendency apparent was for ABS to enhance simultaneous negative contrast at the higher dose levels. Thus, both CDP and ABS tend to reduce successive negative contrast, but either enhance or do not influence simultaneous negative contrast.

The present data may also provide information relevant to understanding the mode of operation of drugs that act to reduce negative contrast. Both CDP and $\mathrm{ABS}$ have been found to reduce negative contrast under specific conditions when administered for the first time postshift. Ethanol has also been found to substantially reduce contrast when administered during the postshift period (Becker \& Flaherty, in press). The effects of ethanol and CDP on contrast are very similar: both have no effect on contrast when administered on the 1st postshift day but both reduce contrast when administered on the 2nd or subsequent postshift days (Becker \& Flaherty, in press; Flaherty et al., 1980; Vogel \& Principi, 1971). The present study and one earlier one (Flaherty \& Driscoll, 1980) indicate that ABS has a different pattern of effectiveness: it tends to reduce contrast on both the 1st and 2nd postshift days. To what might these similarities and differences be related? There is substantial evidence that benzodiazepines, barbiturates, and $\mathrm{EtOH}$ all potentiate the inhibitory neurotransmitter gammaamino-butyric acid (GABA) at the neuronal level (e.g., Nestros, 1980; Skolnick, Paul, \& Barker, 1980; Tallman, Paul, Skolnick, \& Gallager, 1980). A reasonable hypothesis is that all three drugs reduce contrast by potentiating GABA, which acts to inhibit the system that produced contrast in the first place.

One problem with the GABA hypothesis is that ABS has a tendency to reduce contrast on the 1st postshift day whereas the other compounds do not have this tendency. One possible explanation for this difference may be derived from recent studies showing that stereoisomers of barbiturates have opposing effects on the neuronal level (Huang \& Barker, 1980; Mathers \& Barker, 1980).

The ( $(-)$ isomer of pentobarbital has been found to potentiate GABA and to have inhibitory effects at the neuronal level, whereas the $(+)$ isomer has been found to have excitatory effects at the neuronal level. In our experiments, we used a racemic mixture of ABS. If the stereoisomers of ABS have effects similar to those demonstrated for pentobarbital, then the differential effects of ABS on the 1st and 2nd postshift day may be due to different mechanisms. The commonality of the effects of ABS, CDP, and EtOH on the 2nd postshift day may be related to the common GABA-enhancing properties of CDP, EtOH, and the $(-)$ isomer of the barbiturate. The effects of ABS on the 1st postshift day, effects not shared by CDP and $\mathrm{EtOH}$, may be due to the $(+)$ isomer of the barbiturate. All of these speculations are, of course, amenable to experimental analysis.

The present results are also relevant to distinguishing between simultaneous and successive contrast effects. Previous research has shown these two contrast paradigms to be differentially sensitive to CDP (references previously cited), to endure for different periods of time (Flaherty et al., 1977), to be differentially sensitive to the disinhibitory effects of a novel stimulus (Lombardi \& Flaherty, 1978), to differ in the ease with which both may be obtained in runway behavior with shifts in the concentration of sucrose or in the degree of reward delay (Flaherty, Riley, \& Spear, 1973; Mackintosh \& Lord, 1973), and to differ in likelihood of their occurrence in different species of animals (e.g., Pert \& Gonzalez, 1974). The present study adds their differential sensitivity to ABS to this list. The question of why ABS apparently has differential effects on contrast in instrumental behavior occurring in the runway and contrast in consummatory behavior cannot be answered at the present time. 


\section{REFERENCES}

Barry, H., III, \& Miller, N. E. Effects of drugs on approachavoidance conflict tested repeatedly by means of a "telescope alley." Journal of Comparative and Physiological Psychology, 1962, 55, 201-210.

Barry, H., III, Wagner, A. R., \& Miller, N. E. Effects of alcohol and amobarbital on performance inhibited by experimental extinction. Journal of Comparative and Physiological Psychology, 1962, 55, 464-468.

Becker, H. C., \& Flaherty, C. F. Influence of ethanol on contrast in consummatory behavior. Psychopharmacology, in press.

Flaherty, C. F., \& Driscoll, C. D. Amobarbital sodium reduces successive gustatory contrast. Psychopharmacology, 1980, 69, 161-162.

Flaherty, C. F., \& Largen, J. Within-subjects positive and negative contrast effects in rats. Journal of Comparative and Physiological Psychology, 1975, 88, 653-664.

Flaherty, C. F., Lombardi, B. R., Kapust, J., \& D'Amato, M. R. Incentive contrast undiminished by extended testing, imipramine, or chlordiazepoxide. Pharmacology, Biochemistry and Behavior, 1977, 7, 315-322.

Flaherty, C. F., Lombardi, B. R., Wrightson, J., \& Deptula, D. Conditions under which chlordiazepoxide influences successive gustatory contrast. Psychopharmacology, 1980, 67, 269-277.

Flaherty, C. F., \& Meinrath, A. B. The influence of scopolamine on sucrose intake under absolute and relative test conditions. Physiological Psychology, 1979, 7, 412-418.

Flaherty, C. F., Riley, E. P., \& Spear, N. E. Effect of sucrose concentration and goal units on runway behavior in the rat. Learning and Motivation, 1973, 4, 163-175.

Flaherty, C. F., Wrightson, J., Deptula, D., \& Duston, C. Chlordiazepoxide does not influence simultaneous gustatory contrast. Bulletin of the Psychonomic Society, 1979, 14, 216-218.

Huang, L. M., \& Barker, J. L. Pentobarbital: Stereospecific actions of the $(+)$ and $(-)$ isomers revealed on cultured mammalian neurons. Science, 1980, 207, 195-197.

Lombardi, B. R., \& Flaherty, C. F. Apparent disinhibition of successive but not of simultaneous contrast. Animal Learning \& Behavior, 1978, 6, 30-42.
Mackintosh, N. J., \& LoRD, J. Simultaneous and successive contrast with delay of reward. Animal Learning \& Behavior, 1973, 1, 283-286.

Mathers, D. A., \& Barker, J. L. (-) Pentobarbital opens ion channels of long duration in cultured mouse spinal neurons. Science, 1980, 209, 507-508.

Miller, N. E. Some recent studies of conflict behavior and drugs. American Psychologist, 1961, 16, 12-24.

Nestros, J. N. Ethanol specifically potentiates GABA-mediated neurotransmission in feline cerebral cortex. Science, 1980, 209, 708-710.

Pert, A., \& Gonzalez, R. C. Behavior of the turtle (Chrysemys picta picta) in simultaneous, successive and behavioral contrast situations. Journal of Comparative and Physiological Psychology, 1974, 87, 526-538.

Reid, L. D., Wasden, R. E., \& Courtney, R. J. Reinforcing limbic system stimulation and sodium amytal. Psychonomic Science, 1970, 18, 47-48.

RIDGers, A. J., \& Gray, J. A. Influence of amylocarbitone on operant depression and elation effects in the rat. Psychopharmacologia, 1973, 32, 265-270.

Rosen, A. J., Glass, D. H., \& Ison, J. R. Amobarbital sodium and instrumental performance changes following reward reduction. Psychonomic Science, 1967, 9, 129-130.

Rosen, A. J., \& Tesse L, R. E. Chlorpromazine, chlordiazepoxide, and incentive-shift performance in the rat. Journal of Comparative and Physiological Psychology, 1970, 72, 257-262.

Skolnick, P., Paul, S. M., \& Barker, J. L. Pentobarbital potentiates GABA-enhanced $\left[{ }^{3} \mathrm{H}\right]$-diazepam binding to benzodiazepine receptors. European Journal of Pharmacology, 1980, 65, 125-127.

Tallman, J. F., Paul, S. M., Skolnick, P., \& Gallager, D. W. Receptors for the age of anxiety: Pharmacology of the benzodiazepines. Science, 1980, 207, 274-281.

Vogel, J. R., \& Principi, K. Effects of chlordiazepoxide on depressed performance after reward reduction. Psychopharmacologia, 1971, 21, 8-12.

(Manuscript received June 2, 1981; revision accepted for publication December $4,1981$. 